\title{
Erhöhung der Franchise
}

\section{Hans Stalder}

Prof. Dr. med., Mitglied der Redaktion

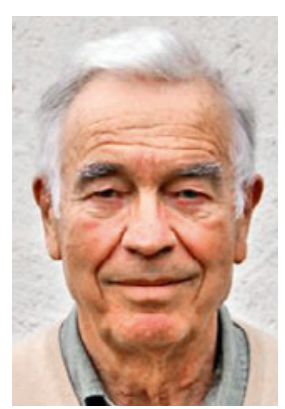

Ende September hat der Ständerat eine Motion zur Erhöhung der Krankenkassen-Franchise gebilligt. Es gibt mindestens drei Gründe, die gegen eine solche Erhöhung sprechen.

1. Auf den ersten Blick erscheint es offensichtlich, dass eine Erhöhung der Franchise die Gesundheitskosten senken müsste, da sie zu einer Verringerung von Konsultationen wegen Bagatellen führen dürfte. Die Situation ist jedoch komplexer, als es den Anschein hat. Eine Arbeit mit fast 900000 Patienten in 36 US-Gesundheitsplänen [1] machte deutlich, dass eine Erhöhung des Selbstbehalts der Patienten (oder die Einführung eines solchen) in der Tat zwar die Anzahl der ambulanten Behandlungen senkt - was wiederum zu einer Senkung der ambulanten Kosten führt -, jedoch die Gesundheitskosten insgesamt erhöht, da die Reduzierung der ambulanten Behandlungen zu einem Anstieg der Hospitalisationen führt. Vergleichbare Ergebnisse liessen sich auch in anderen Gesundheitssystemen finden, beispielsweise in Osteuropa oder in Entwicklungsländern. In Grossbritannien, wo es keinerlei Selbstbeteiligung an den Gesundheitsleistungen gibt, sind die ambulanten Behandlungen erwartungsgemäss häufiger als in der Schweiz. Die Anzahl der Hospitalisationen pro Einwohner ist aber deutlich niedriger [2], was zumindest teilweise den grossen Unterschied in den Gesundheitskosten der beiden Länder erklären könnte.

2. Wenn also die Erhöhung der Franchise wirtschaftlich umstritten ist, sind ihre Auswirkungen deutlich schwerwiegender. Die Reduzierung der ambulanten Behandlungen verteilt sich nämlich nicht gleichmässig auf die Bevölkerung. Vermögende Personen werden die ambulante Behandlung weiterhin in Anspruch nehmen, während der schlechter gestellte Teil der Bevölkerung darauf verzichtet [1]. In der Schweiz ist dies bereits der Fall [3]. Zudem ist bekannt, dass jene, die sich aus wirtschaftlichen Gründen nicht mehr ambulant behandeln lassen, häufiger an chronischen Erkrankungen leiden, als jene, die zum Arzt gehen [1, 3]. Die Erhöhung der Franchise trifft also vor allem diejenigen, die es am nötigsten hätten, behandelt zu werden: die wirtschaftlich schwachen und kranken Personen.
3. Der FMH-Präsident erläuterte jüngst, warum die Krankenkassenbeiträge stärker steigen als die Gesundheitskosten. Die Kosten für die Hospitalisation werden vom Staat mitgetragen, während die tendenziell stärker steigenden ambulanten Behandlungskosten allein von den Kassen bezahlt werden [4]. Letztere haben daher ein Interesse daran, ambulante Behandlungen in Richtung Hospitalisationen zu verschieben. Eine Erhöhung der Franchise, die zwar die ambulanten Kosten senkt, die Kosten der Spitalbehandlungen jedoch erhöht, kann daher als Versuch der Kassen interpretiert werden, den Prämienanstieg zu dämpfen. Die steigenden Gesamtkosten sollen vor allem die Patienten und der Staat tragen! Da stellt sich die Frage, ob es ein Zufall ist, dass der Initiator der Motion und derjenige, der sie einbrachte, nämlich der Präsident der Gesundheitskommission des Ständerates, Verwaltungsratsmitglieder der Groupe Mutuel bzw. der CSS sind ...

Apropos: Wissen Sie, dass sich die Groupe Mutuel bei ihrer Gründung im Jahr 1890 «Verband der gemeinnützigen Hilfsvereine des Kantons Wallis, SSM» nannte, dessen Mitglieder sich zusammenfanden, um «die Folgen wirtschaftlicher Schicksalsschläge besser abmildern zu können»? Und dass die CSS Versicherung 1899 unter dem Namen «Krankenkasse des katholischen Arbeiterverbandes St. Gallen» entstand als eine christlich geprägte Selbsthilfeorganisation? Sie schützte ihre Mitglieder durch die Rechtsform eines Verbandes vor den wirtschaftlichen Folgen von Krankheit und Unfall. Tempora mutantur ...

Literatur

1 Trivedi AN, Moloo H, Mor V. Increased ambulatory care copayments and hospitalizations among the elderly. NEJM 2010;362:320-8.

2 OECD (2015), «Hospital discharge rates» (indicator). DOI: http://dx.doi.org/10.1787/5880c955-en

3 Bundesamt für Statistik BFS. Gesundheitszustand, Armut und Verzicht auf Pflegeleistungen. Erhebung über die Einkommen und die Lebensbedingungen (SILC) 2011. Neuenburg 2013.

4 Schlup J. Warum steigen die Prämien stärker als die Kosten? Schweizerische Ärztezeitung | Bulletin des médecins suisses | Bollettino dei medici svizzeri | 2016;97:35. 